\title{
Desmoid Tumor Mimicking Uterine Leiomyoma
}

Richa Singh, Vanita Vaishnav, Sriram Gopal, Rajshekhar Yadav

From the Department of Obstetrics \& Gynaecology, Dr. D.Y.Patil

Hospital, Sector 7, Nerul, Navi Mumbai, Maharashtra, India.

\section{Abstract:}

Desmoid is a rare tumor caused by abnormal proliferation of fibroblastic cells. The tumor may arise from fascia or/and aponeurosis of muscle. They may reach huge sizes often causing a diagnostic dilemma. Here, we present a rare case of desmoid tumor mimicking broad ligament fibroid, in a 23 year old woman without any known risk factors. Initial investigations attributed to wrong diagnosis of uterine leiomyoma whereas further investigations and surgical exploration revealed it to be a desmoid tumor. The tumor was removed completely with clear margins, and the decision regarding adjuvant chemo/radiotherapy was kept on hold depending upon the recovery and follow up visits. The patient has remained asymptomatic till date. In the case reported here, the patient's only presenting complaint was a painless abdominal mass. This added to the initial difficulty in diagnosis. So, thorough clinical examination keeping in view all the possible presentations of an abdominal mass in women is equally important in all such cases. Residual tumor and recurrence after initial resection may require adjuvant treatment with chemotherapy and/or radiation in addition to repeat surgery. Close follow up of the patient is the key to complete recovery.

Key words: Neoplasms, Broad ligament, Leiomyoma, Gardner syndrome, Chemotherapy.

\section{Introduction}

Desmoid tumor is a rare type of cancer that develops in the tissues that form tendons and ligaments, usually in the arms, legs or abdomen and sometimes in the chest. These tumors, also called aggressive fibromatosis, are considered benign because they typically don't spread to other parts of the body. They may invade nearby tissues and organs, however, and can be difficult to control.

Although the cause of these tumors is not known, researchers have found that they tend to occur in people who have a family history of polyposis coli, a hereditary colon cancer. The synonym aggressive fibromatosis describes the marked cellularity and aggressive local behavior. This course and the tendency for recurrence makes the treatment of these relatively rare fibrous tumors challenging. Treatment options include surgery, chemotherapy and radiation, depending on the location of the tumor and patients' age. We hereby report 23 year old women with desmoid tumor with presentation mimicking that of broad ligament fibroid.

\section{Corresponding Author: Dr. Vanita Vaishnav}

Email: drvanitadhar@gmail.com

Received: August 17, 2013 | Accepted: September 17, 2013 | Published Online: September 25, 2013 This is an Open Access article distributed under the terms of the Creative Commons Attribution License (creativecommons.org/licenses/by/3.0)

Conflict of interest: None declared | Source of funding: Nil | DOl: http://dx.doi.org/10.17659/01.2013.0079 


\section{Case Report}

23 years old married woman, para 2 living 2, was referred to our hospital from a primary health centre, with history of a gradually increasing painless mass in left iliac and left lumbar region since three years. There was no history of any bowel, bladder or menstrual complaints. Ultrasound (USG) and Computed Tomography (CT) scan done elsewhere were suggestive of broad ligament fibroid. On clinical examination, the patient was of average height and build. General examination was unremarkable. On abdominal examination, a huge, hard immobile mass with irregular margins was palpable in left lower quadrant extending to pelvis, measuring approximately $25 \times 20 \mathrm{~cm}$ [Fig.1]. Repeat CT scan of the abdomen-pelvis with intravenous contrast showed a large well defined lobulated soft tissue density mass measuring $25.0 \times 17.0 \times 13.0 \mathrm{~cm}$ in pelvis, displacing the urinary bladder posteriorly and to the right [Fig.2]. Anteriorly it was abutting the abdominal wall. The fat planes of anterior abdominal wall were disturbed. It was suspected to be due to broad ligament fibroid. For better definition of this soft tissue mass, MRI was done. It showed a heterogeneously enhancing mass arising from the abdominal wall, iso-intense to low signal on T1- and heterogeneously high on T2weighted images. There was no visceral or lymph node involvement. Bilateral ovaries were found to be normal on CT as well as MRI. Thus provisional diagnosis of desmoid tumor was made.

The mass was explored with a left paramedian incision. Division of anterior rectus sheath revealed a large solid mass, arising from the oblique muscles and occupying the entire extra-peritoneal space, extending till sacral promontory. The tendinous base of the mass was attached to the inner margin of iliac crest. Despite of its size, the mass was relatively avascular allowing complete surgical excision without much difficulty [Fig.3]. The abdominal wall reconstruction was done by double breasting the

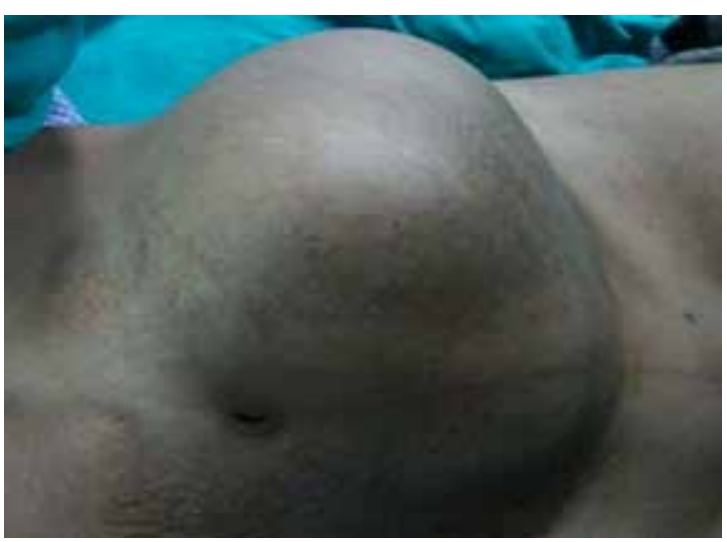

Fig.1: Clinical presentation of the patient with large hard fixed abdominal mass.

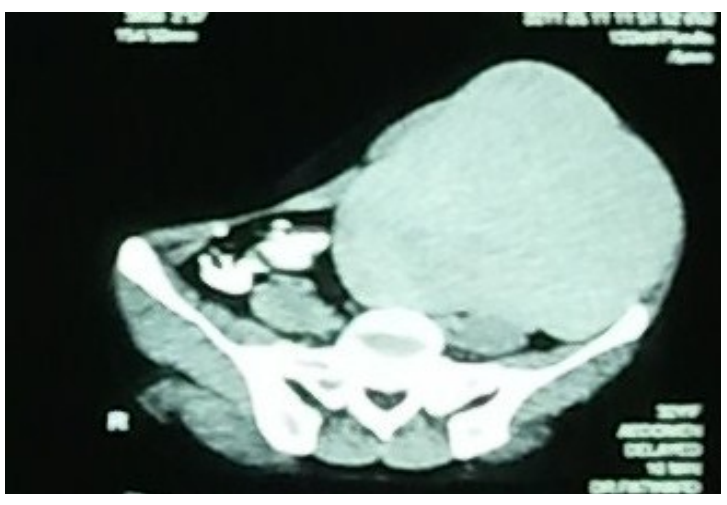

Fig.2: CT scan showing the lesion.

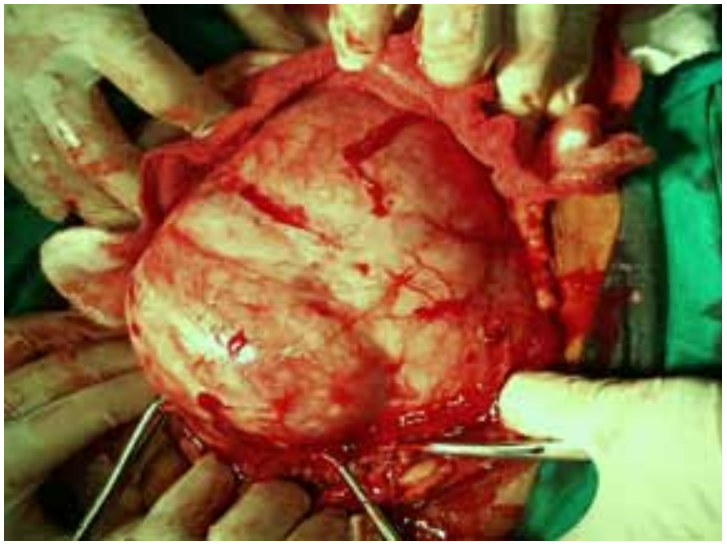

Fig.3: Intraoperative finding: Completely excised tumor mass. 
redundant anterior rectus sheath. Post-operative period was uneventful. Histopathologic examination confirmed the diagnosis of desmoid tumor [Fig.4]. At six weeks follow up, the surgical wounds healed completely and the patient was asymptomatic. Patient is kept under regular long term surveillance plan.

\section{Discussion}

Desmoid tumors are cytologically bland fibrous neoplasms originating from the musculo-aponeurotic structures throughout the body. The term Desmoid, coined by Muller in 1838, is derived from the Greek word desmos, which means tendon-like.

Although desmoid tumors most commonly arise from the rectus abdominis muscle in postpartum women and in scars due to abdominal surgery, they may occur in any skeletal muscle. The cause of desmoid tumors is uncertain. It may be related to trauma, genetic or hormonal factors. The case reported here was sporadic with no positive family history as well as no history of trauma/surgery. Desmoid tumors tend to infiltrate adjacent muscle bundles, frequently entrapping them and causing their degeneration. They may be derived from mesenchymal stem cells [1]. Although fixation to musculo-aponeurotic structures is apparent, the overlying skin remains normal, as was found in the reported case.

Gardner syndrome or familial adenomatous polyposis (FAP) is characterized by colorectal adenomatous polyps and soft tissue and bony neoplasms. Gardner syndrome was regarded as a separate disease until the identification of the APC (adenomatous polyposis coli) gene, at which point mutations in the APC gene were recognized as the underlying cause of both Gardner syndrome and FAP. Desmoid tumors occur at a rate of $10-15 \%$ in patients with FAP, an autosomal recessive inherited disease caused by germ line mutations in the APC gene. Sporadic forms have no hereditary background.

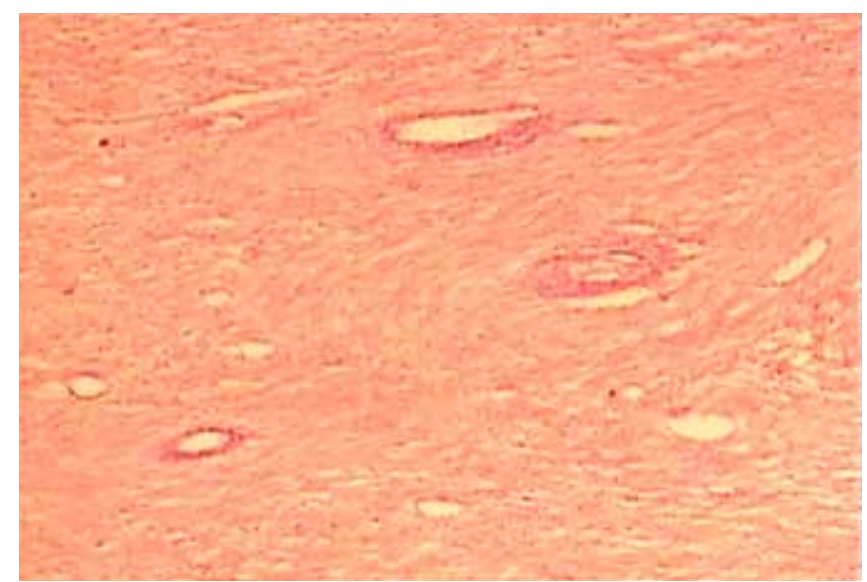

Fig.4: Microscopy: Spindle cells surrounding muscle cells.

Overall, desmoid tumors account for $0.03 \%$ of all neoplasms. Incidence is as high as $13 \%$ in patients with familial polyposis of the colon [2]. Despite their benign histological appearance and negligible metastatic potential, the tendency of desmoid tumors to cause local infiltration is significant in terms of (i) deformity, morbidity, and mortality resulting from pressure effects and (ii) potential obstruction of vital structures and organs. Although desmoid tumors are more common in persons aged 10-40 years than in others, they do occur in young children and older adults. It commonly develops in the anterior abdominal wall and shoulder girdle. A history of trauma (often surgical) to the site of the desmoid tumor is elicited in 1 in 4 cases [3].

Intra-abdominal desmoid tumors remain asymptomatic until their growth and infiltration cause visceral compression. Although our patient had a large mass, there were no pressure symptoms and the only presenting complaint was painless enlarging abdominal mass. Symptoms of intestinal, vascular, ureteric, or neural involvement may be the initial manifestations. The natural history of desmoids varies from patient to patient, ranging from unceasing growth to spontaneous regression. 
A large desmoid tumor may cause diagnostic dilemma. Commonly encountered large pelvic masses in women are fibroids, dermoid cysts, ovarian cysts and ovarian cancer [4]. However, uncommon pelvic masses such as mesothelioma, adenosarcoma, leiomyosarcoma and desmoid tumor should be considered in differential diagnosis [5]. Even USG and CT scan may incorrectly attribute a large abdominal wall desmoid tumor to leiomyoma. In the case presented, sheer size and extent of tumor made it very difficult to ascertain the site of origin and lead to initial incorrect diagnosis of broad ligament fibroid.

CT scanning and MRI are used for the diagnosis and follow-up of desmoid tumors. They can help determine the extent of the tumor and its relationship to nearby structures, especially prior to surgical removal. MRI is superior to $\mathrm{CT}$ scanning in defining the pattern and the extent of involvement as well as in determining if recurrence has occurred after surgery. There have been studies regarding the clinical features that differentiate desmoids from Gardner's tumor. One such study found that desmoid tumors associated with Gardner's syndrome were more likely to be multiple $138 \%$, five of 13 patients) and to involve the mesentery $(60 \%, 18$ of 30 tumors) and the abdominal wall (40\%, 12 of 30 tumors), whereas isolated desmoid tumors were singular (all 10 patients) and were located in the retro peritoneum (six cases), pelvis (three), and anterior wall (one). Desmoids related to Gardner's syndrome also tended to be smaller (mean diameter, $4.8 \mathrm{~cm}$ ) than idiopathic desmoids (mean diameter, $13.8 \mathrm{~cm}$ ). Desmoid tumors associated with Gardner's syndrome tend to occur in the mesentery and abdominal wall, whereas isolated desmoids involve the retro peritoneum and pelvis [6].

Biopsy of the mass provides absolute diagnosis and one can choose between a fine-needle aspiration, incisional and excisional biopsy techniques depending on the site and size of the mass. Because the tumors cannot metastasize, there may be a role for observing the patient if the lesion is small and not causing functional difficulty.

Surgical treatment consists of aggressive, complete wide resection. Large defects in anterior abdominal wall as a result of radical resection are managed with immediate mesh reconstruction to minimize functional disability [7].

Local desmoid tumor recurrence rates are reported to be as high as $70 \%$. Positive margins after surgery reflect a high risk for recurrence. Residual tumor and recurrence after initial resection may require adjuvant treatment with chemotherapy and/or radiation in addition to repeat surgery. Radiation therapy may be used as a treatment for recurrent disease or as primary therapy to avoid mutilating surgical resection. It may be used postoperatively, preoperatively, or as the sole treatment [8]. Pharmacologic therapy with antiestrogens and prostaglandin inhibitors may also be used.

In this case, the tumor was removed completely with clear margins, and the decision regarding adjuvant chemo/radiotherapy was kept on hold depending upon the recovery and follow up visits. The patient has remained asymptomatic till date. Since desmoid tumors are rare, all cases should be reported for better understanding of this complex disease.

\section{Conclusion}

Although desmoid tumor is a rare entity, it should always be kept in mind while examining a patient with abdominal mass or swelling. Radiological investigations like USG, CT scan, MRI are helpful in differentiating desmoid from other masses, but they can be misleading at times. Therefore, clinical assessment of the patient including detailed history and examination is of utmost importance for accurate diagnosis. 


\section{References}

1. Wu C, Amini-Nik S, Nadesan P, Stanford WL, Alman BA. Aggressive fibromatosis (desmoid tumor) is derived from mesenchymal progenitor cells. Cancer Res. 2010;70:7690-7698.

2. Klemmer S, Pascoe L, DeCosse J. Occurrence of desmoids in patients with familial adenomatous polyposis of the colon. Am J Med Genet. 1987;28:385-392.

3. Lopez R, Kemalyan N, Moseley HS, Dennis $D$, Vetto RM. Problems in diagnosis and management of desmoid tumors. Am J Surg. 1990; 159:450-453..

4. Errarhay S, Mamouni N, Mahmoud S, El fatemi $H$, Saadi $H$, Mesbahi O. Primary Malignant Melanoma of the Female Genital Tract: Unusual Localization. Journal of Case Reports. 2013;3:169-175.
5. Ichikawa T, Koyama A, Fujimoto $H$, et al. Abdominal wall desmoid mimicking intraabdominal mass: MR features. Magn Reson Imaging. 1994;1 2:541-544.

6. Kawashima A, Goldman SM, Fishman EK, Kuhlman JE, Onitsuka H, Fukuya T, et al. CT of intraabdominal desmoid tumors: is the tumor different in patients with Gardner's disease. Am J Roentgenol. 1994;162:339-342.

7. Bertani E, Chiappa A, Testori A, et al. Desmoid tumors of the anterior abdominal wall: results from a monocentric surgical experience and review of the literature. Ann Surg Oncol. 2009; 16:1642-1649.

8. Constantinidou A, Jones RL, Scurr M, Al-Muderis $O$, Judson I. Advanced aggressive fibromatosis: Effective palliation with chemotherapy. Acta Oncol. Aug 30 2010; 50:455-461. 